\title{
Research and Application of China Well Information System Based on ArcIMS
}

\author{
Huafeng Xie (corresponding author), Jinyin Yin, Ming Li, Xin Liu \& Baojiang Zhang \\ Research Institute of Petroleum Exploration and Production \\ SINOPEC, Beijing 100083, China \\ E-mail: raobian@126.com \\ Muwei Chen \\ Research Institute of Petroleum Exploration and Production \\ Petrochina, Beijing 100083, China
}

\begin{abstract}
Well data is the important and basic data for geology work. In order to develop and utilize well information resources more efficiently, the present paper attempted to use ArcIMS 9.0 as development platform to realize functions of WebGIS, based on $\mathrm{C} / \mathrm{S}($ Client/server) or B/S(Browser/server), utilize ASP(active server page) and ADO (active data object) to write codes for updating and maintaining attribute data, achieve share and exchange of well data on internet in combination with ActiveX. At the same time, the present paper resolved the problem of Chinese display.
\end{abstract}

Keywords: ArcIMS, ActiveX, Well data, WebGIS, Internet

\section{Introduction}

Currently, management mode of well data has been relatively passive and backward, and storage medium of part data have always been paper carrier or data entity. Works on the archives information processing have been less developed, and there have been great limitations on the preservation, utilization and service. Information was less available, and tough to lookup with low efficiency and difficulty to manage and maintain. It was tough to meet the requirement of open and diversified development on the exploitation and utilization of well data imposed by users, which affected the utilization of file data. Well file data is significant information, and important basis for the planning, management and rational utilization of all kinds of well. A specific geological work, was always to investigate all existed geological data at large, and finally form new geological data, again and again, and it would become huge social wealth of human being(Xin, 2008). Additionally, well data possessed the characteristics of high cost, large quantity, complicated types, huge potential of social and economic benefits, and have been extensively applied in the areas such as earth science, mineral investigation and development, environmental protection and so on. Well file data was precious information resources, and should be promptly undertaken development and utilization.

Along with the development of geographic information system(GIS) and database technology, especially the convenience of ArcIMS on establishing atlas, developing web pages with links to atlas and managing map website, and the application of its architecture and function in publishing maps, data and meta data, all afforded the likelihood of realizing the united storage and visual management of well file data(2004). In the present paper, we combined the strong advantages of ArcIMS and ASP, aiming at the practical situation of well data of geological industry, and devised a set of concise and efficacious data management scheme. According to the well attribute data and vector base map files, Webgis navigation main interface was produced; Code was written by ASP, and data transmitted from Webgis navigation main interface was used to realize the query, search and orientation of geological data. According to the security requirements and degree of importance, relative data was used by the combination of $\mathrm{C} / \mathrm{S}$ and $\mathrm{B} / \mathrm{S}$.

\section{System overall design}

\subsection{Outline}

This system included parts of database server and client application. Database server mainly realized the untied storage 
of spatial data and attribute data, among which spatial data contained geographic base map and well position chart, and attribute data included static and dynamic information. Client part belonged to data updating system, used as the maintenance and application of data.

\subsection{Overall structure design}

This system adopted the development mode with the combination of $\mathrm{C} / \mathrm{S}$ and $\mathrm{B} / \mathrm{S}$, and could be disposed to different system structure according to the importance degree of well file data. $\mathrm{C} / \mathrm{S}$ reasonably allocated assignments to the client and Sever, and made up the deficiency of ASP in security. In combination with the object of ASP with no necessary compiling, without problems of browser compatibility, component function of ActiveX Server could be extended, and database access could be convenient (using ADO component) with advantages of hiding program code and convenient compiling(Luo,2004, PP.58-62). It lowered the expenditure of system communication, and a host of work could be submitted to server after processing in client, which exerted the processing capability of client and embodied the characteristics of prompt response in lan.

$\mathrm{B} / \mathrm{S}$ was a supplement and amelioration for $\mathrm{C} / \mathrm{S}$, and user interface carried out through browsers, which alleviated the workload and cost of system maintenance and updating. In combination with the advantages of $\mathrm{C} / \mathrm{S}$, such as security, celerity and nicety in Lan, and those of $\mathrm{B} / \mathrm{S}$, such as opening, convenience, information share in high grades and so on, server was decomposed into data server and application server, forming the architecture with three layers, which was depicted in Figure 1. B/S could differentiate data access operation and application model, using ArcSDE as the GIS application server in C/S, IIS as Web server, through the data filtering of server side, less network traffic was obtained with rapid execution speed. In combination with better system structure of ArcIMS, information transfer mechanism, and organization and spatial distribution of multi-source data, stability and efficiency of the whole system was guaranteed(Li, 2003).

\subsection{Function design}

Data updating system: main objective was to manage well file data, and through data updating, renovate and maintain well information and corresponding file data at any moment, keeping the real-time of information.

Application system: application function such as inquiring about, calculating, browsing and downloading well file data.

General function: general functions GIS possessed such as zooming in and out and rambling the atlas, data location and lookup. As well as "eagle-eye" function, namely that index map in the indicated map of atlas frame. Users could easily know the relationship between the present map and panorama, and immediately locate their concerned positions.

\section{Key technology}

\subsection{WebGIS}

Main technical methods such as CGI(Common Gateway Interface) and browser plug in for WebGIS had limitations, for instance zoom-in and -out of client images were tough, and data excessively fasten on the client. ActiveX was the standard developed with attempts to adapt, based on OLE(Object Linking and Embedding) standard, and offered public frame for extending Internet Explorer function of Microsoft Web. ActiveX was a software module used for accomplishing certain assignment and information communication, processing GIS data and completing GIS analysis, and could be used by any programming languages or application system supporting OLE standard(Rohit, 2003). ActiveX was one of the four customization procedures ArcIMS provided, and ActiveX connector was used to link web server and ArcIMS application server. Map could be added to ASP or Visual Basic, and based on Internet GIS ActiveX, processing and display of GIS data were done depending on GIS ActiveX. ActiveX closely hanged together with web browser flexibly and seamlessly. Under the normal condition, GIS ActiveX was contained in the HTML codes, and obtained through the reference label of $<\mathrm{OBJECT}>(2003)$.

ASP have substituted CGI, ISAPI, JDBC and so on due to its dynamic high efficiency and easy alteration. It not only combined HTML pages, Scripts language and dynamics server component, but also made all processing accomplished in the server. Additionally, the output of ASP files was suitable for the HTML of Web browser. HTTP(Hypertext Transfer Protocol) adopted stateless mechanism in order to enhance work efficiency of server, but had no memory capability for transaction process, which caused that each connection needed more information transmitted and attenuated work efficiency to some extent. Therefor, when developing system ASP, such problem could be solved by ASP implicit object Session. Taken together, we implemented WebGIS technology by the combination of ASP and ActiveX in the present paper(Rohit).

\subsection{Multi-source and distributed database technology}

ADO technology linked general relational database into the system, extended data source for system, and afforded broader data support for making thematic maps. Its cardinal advantages were easy to operate, high speed, low memory expenditure and less occupied disk space. Moreover, it had the function of remote data service(RDS), transmitted data from server to client application procedure or Web pages during one two-way process by RDS, implemented data 
process in client and then return the updated information to server. As for setting system user authority and designing query module, it applied the combination of ASP and database access technology typically. ArcSDE(Spatial Database Engine) was GIS channel between ArcGIS and relational database, and geological database server on the basis of relational database. Application of ArcSDE could realize seamless integration of spatial data and attributed data, which could not only accelerated the query of system, but also facilitated managers to manage and maintain database. Accordingly, we applied the technological mode of Access + ArcSDE, which could store spatial data, attributed data and well file information in different servers, erect multi-source database. Data transmission bottleneck would not occur just due to excessive dependence on one server. It would form a flexible data system, which offered technology guarantee for distributed global share(Liu, 2009).

\subsection{Chinese display technology}

Due to operating system version and damage and deletion of related files, character sets of server and client differed, and Chinese display in ArcIMS was irregular. ArcIMS atlas service customization, management and issued software were all based on the function of JRE(Java Runtime Environment). During running, when JRE couldn't parse Chinese, Chinese fonts in softwares such as Author were disorderly codes or no display, and thus JRE Chinese display was required to collocate(2008). One more situation, Chinese character sets of database didn't match those of ArcIMS, for data was read from database, and thus Chinese display in ArcIMS could be also irregular. Manifestation and solution were as follows:

3.3.1 Disorderly codes in web pages(Chinese display of ArcIMS in html viewer was disorderly codes), and codes were "?", panes, fonts similar to Chinese-traditional characters and so on. Occurred positions were mainly filed name, and layers and lists of query. Solutions were as follows:

A. Edited aimsXML.js file in the working directories of javascript. Replaced var charSet="ISO-8859-1"by var charSet= one certain Chinese character sets. For example: var charSet="GB2312".

B. Edited mapFrame.htm file in the working directories of viewer.htm. Edited the following line: <meta http-equiv="Content-Type" content="text/html; charset=ISO-8859-1" $>$. Replaced the previous described ISO-8859-1 by character sets required, such as GB2312.

C. Acquiescently, ArcIMS HTML Viewer used Arial font. In order to display fonts exactly, edited corresponding part of ArcIMSParam.js file:var legFont="Arial"; Replace Arial by corresponding system fonts, such as var legFont="blackbody".

3.3.2 Chinese label disorder codes in atlas, and main codes were panes which appeared in atlas(namely pictures returned from ArcIMS server). Solutions were as follows:

A. Checked the letters and found out whether they were variable transmitted from page inner to set up a dynamic layer. If so, see whether those letters during the period of transfer were normal, or the issue was coding.

B. If not, examined the fonts of these disorder code fields of the map using designer. Looked up in the font directories of corresponding computers, and found out whether there was such fonts or not. Issues occurred due to such fonts.

C. Installed the font, or replace it by a font already existed in your computer.

Excluding approaches of setting character sets to make Chinese information normal, there was still another way to attained such goal, such as chinesization of ArcIMS. Firstly, downloaded chinesization files of ArcIMS in ESRI Chinese website instead of corresponding files. Mainly five modules for chinesization were Manager, Viewer, MetadataExplorer, Esriadmin, management tool application program and ArcExplore. Management tool application program included ArcIMS Administrator, ArcIMS Author and ArcIMS Designer.

\section{Function realization}

\subsection{Multi-source database}

Database was the basis of the present system, including attribute and spatial database. According to the situation, these data could be disposed at different servers, which was in more favor of data update and transmission efficiency.

Information of attribute database in relation to well archives data were mainly well coordinate information, well basic information, well sort information, well data information and well data sort information.

Spatial database used 1:2,500,000 Chinese provincial administrative map as backing, and was published using shapefile format. Map layers published included Chinese administrative division base map, Chinese coast map with provinces, Chinese maritime space map, Chinese main sea area map and Chinese well location map. Users could browse and inquire about properties of layers through activating operation, and put these data through the modules of ArcIMS management tool such as Author, Administrator and Deginer to process, which could possibly form web pages. For easy display, part content was selected to give a demonstration. 


\subsection{Data acquisition}

GIS database server located at the back end of prototype system, and was storage base of system original geological information, with functions of managing GIS original data, responding the request imposed by ASP engine and sending generated HTML pages to WebGIS server(IIS). ArcIMS made well attribute and spatial data formed corresponding WebGIS web pages and files, and modified the generated related files(consulting 3.3 Chinese display technology), which made system able to identify Chinese characters. Opened the hyperlink function, clicked on wells in WebGIS pages(Dave, 2004), used well name as query condition, and then corresponding ASP pages ejected. Just like this, we could manage and apply archives data in these pages. Codes were as follows:

4.2.1 The following codes were the well names to take out and click when WebGIS pages shifted to ASP.

url=Request.ServerVariables("SCRIPT_NAME") 'Return to relative path of present WebGIS page

SearchChar $=$ StrReverse(url) 'Return to the character string in opposite systematic arrangement of relative path

MyPos = InStr(1,SearchChar,"/",1)

'Acquire the character length of well nameif mypos $>1$ then

mypos=mypos -1

'Acquire the wall names by each character

end if

strl= Right(url, MyPos)

'Well name, as the query condition of ejecting ASP pages

4.2.2 The following code was to link and open well archives information base.

Set conn = Server.CreateObject("ADODB.Connection")

DBPath = Server.MapPath("Well.mdb")

conn.Open "driver $=\left\{\right.$ Microsoft Access Driver $\left.\left({ }^{*} . \mathrm{mdb}\right)\right\} ; \mathrm{dbq}=" \&$ DBPath

\section{Conclusions}

The present paper introduced the design and application of Chinese well information system based on ArcIMS. On the basis of ArcADE and Access, we set up multi-source database, and realized the application and management of well data information using ASP, ActiveX, ADO and so on under the premise of a host of data share. According to security and practical requirement, $\mathrm{C} / \mathrm{S}$ or $\mathrm{B} / \mathrm{S}$ could be applied, which realized the unified integrative display of multi-source spatial data and functions of graphics interaction query and access between vector and raster data under the network environment. This intuitive and efficacious interactive visual environment was of great significance to the visual share of complex multi-source spatial data.

\section{References}

ArcIMS ${ }^{\circledR}$. (2004). 9 Architecture and Functionality. An ESRI ${ }^{\circledR}$ White Paper. May 2004.

ArcIMS. installation and collocation. (2008). [Online] Available: http://www.97sky.com/bbs/viewthread.php?tid=260, 2008,10 .

Customizing ArcIMS ActiveX Connector, ESRI. ( 2003). Redlands CA ESRI Press.

Dave, J. (2004). MCAS Miramar GIS Web Site Notes, [Online] Available: http://arcscripts.esri.com/details.asp?dbid=13461, May 03, 2004.

Li, Y., Liu, X.F. (2003). Developing method and research of WebGIS system based on ASP technology. Geo-spatial Information, $2^{\text {rd }}$ issue.

Liu, H.T., \& Du, Y.Y. (2009). Integration and Information Services of Multi-source Spatial Data in Coastal Zone Based on ArcIMS. Journal of image and graphics, $1^{\text {st }}$ issue.

Luo, Y.F. (2004). System for scientific literature's spatial expression and web searches based on ArcIMS. Geo-information Science, 6(2):58-62.

Rohit, P., Carl, L. (2003). Developing Dynamic GIS Web Applications. Department of Computer Science, University of Nevada, Reno.

Rohit, P., Robert, E., \& Franco, B. Programming ArcIMS ActiveX connector with ASP for different browsers/platforms. Biological Resources Research Center,University of Nevada, Reno, NV 89557.

Xin, J.S. (2008). Discussions on the geological data management and socialized service. Land and resources information, $11^{\text {th }}$ issue. 


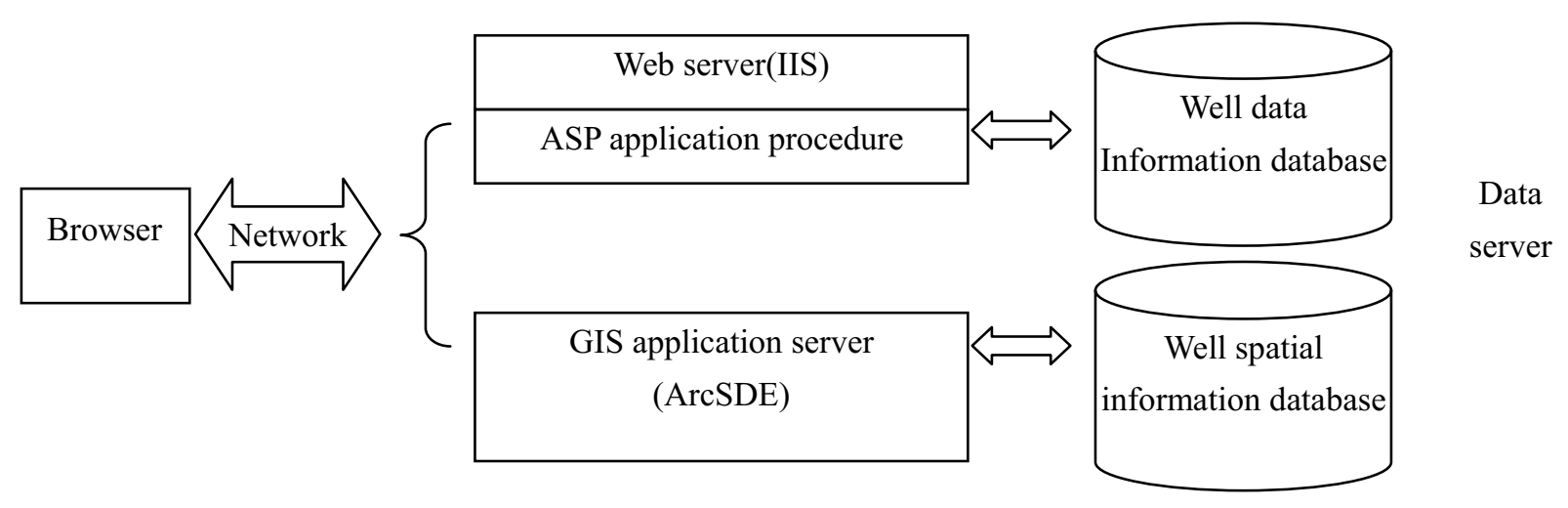

Figure 1. Overall architecture

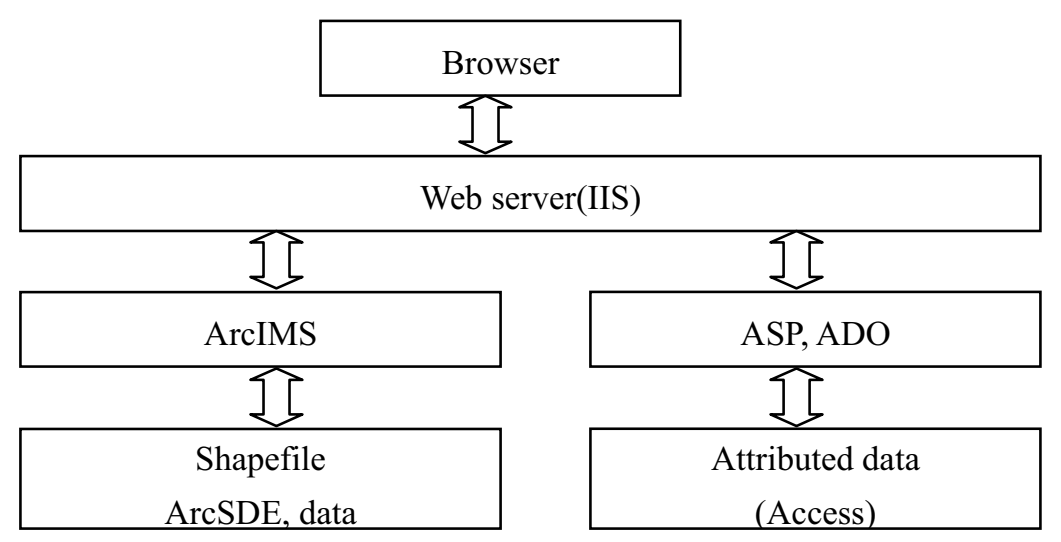

Figure 2. Database schematic diagram 


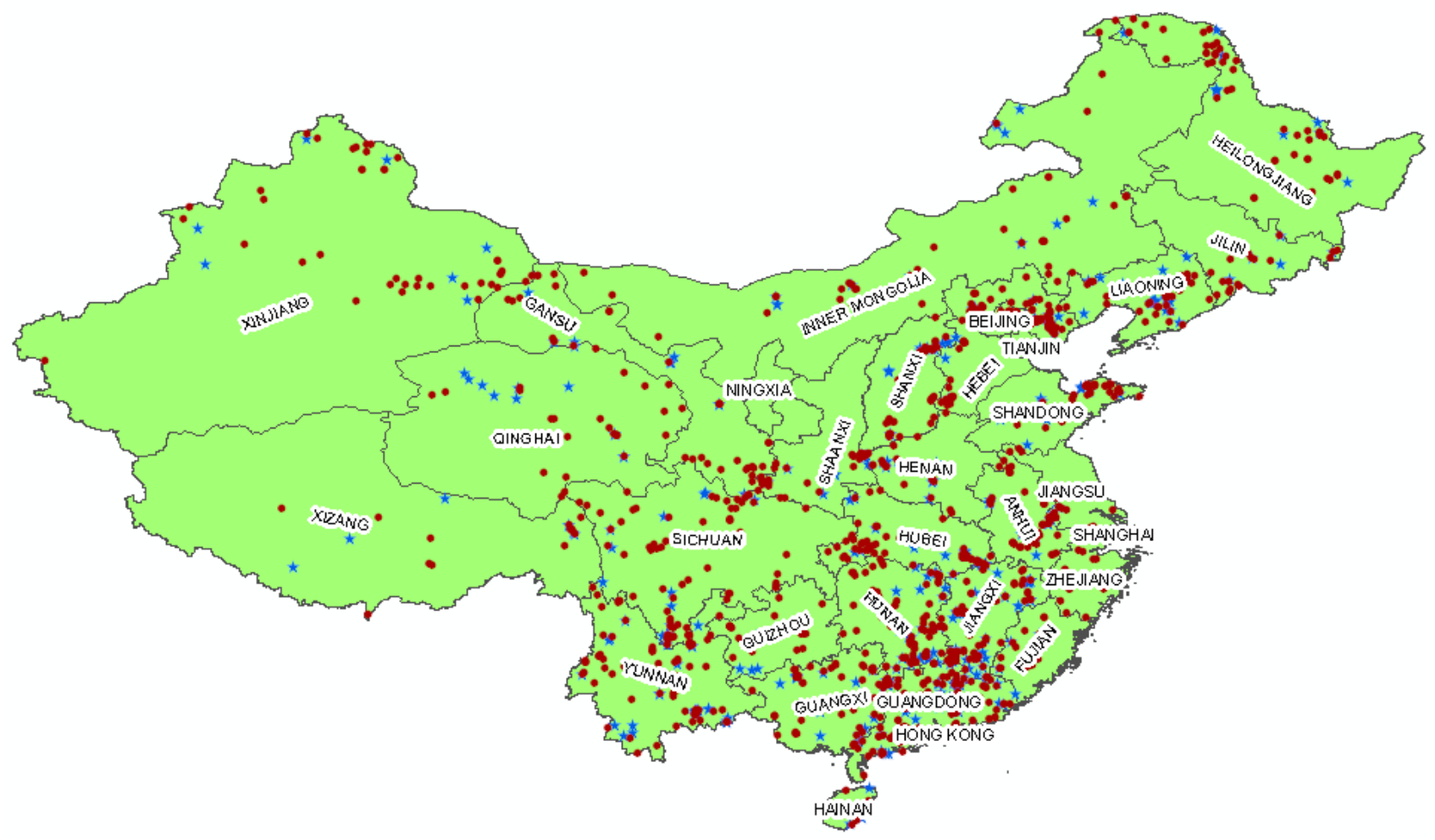

Figure 3. Well location distribution map 